\title{
Novel drug delivery systems, devices, and fabrication methods
}

\author{
Vivek Agrahari ${ }^{1}$ \\ Published online: 4 December 2017 \\ (C) Controlled Release Society 2017
}

Novel therapeutic approaches such as nanotechnology and their innovative fabrication methods have opened a new direction in biomedical sciences. Owing to their small particle size and various other characteristics such as capability of encapsulating small molecules as well as biologics, protecting the drugs in biological environments, improving the drug biodistribution, opportunity for providing controlled or stimuli-responsive drug release, enhancement of the targeted and intracellular delivery of therapeutics, flexibility to be combined with diagnostic and imaging agents for theranostic uses, nanotechnology-based formulations are versatile systems for various applications and for better therapeutic outcomes [1]. The special issue "Novel Drug Delivery Systems, Devices, and Fabrication Methods" covered the recent therapeutic application and development approaches of formulation systems to improve the drug delivery potential. This issue has assembled leaders in drug delivery research areas, covering topics on microparticles, microbubbles, nanoparticles, microneedles, organogels, micelles, lipid-based systems, inorganic nanoparticles, laser-generated micropores, and nanoemulsion. Although, enormous research efforts are being performed into the development of these novel products for various indications, translating these drug delivery systems into a commercial product is challenging [2]. Hence, papers in this issue also discuss about the challenges and approaches to facilitate the translation of delivery systems.

In general, the acceptance of a pharmaceutical product is closely related to the success of the innovation, both commercially and therapeutically. When developing a new technology for drug delivery, consideration must be given to the patient who will be using it. By engaging the patients early in the development process, potential issues can be identified, ensuring the greatest benefits from the product. Microneedles are

Vivek Agrahari

vahw2@mail.umkc.edu

1 School of Pharmacy, University of Missouri-Kansas City, Kansas City, MO 64108, USA novel transdermal drug delivery platforms; however, for a successful translation, it is important to consider the optimal therapeutic outcomes of microneedles by older people. The first article of this special issue by Quinn et al., investigates the insertion parameters in aging skin, together with the feasibility and acceptability of the technology in people aged over 65 years [3]. The work collectively provides the first convincing report of the importance of further translational research of microneedles, ensuring an age-appropriate delivery platform, and strong evidence for future clinical studies. The next article in this special issue by Song et al. investigates the feasibility of transdermal delivery of human growth hormone (hGH) through laser-microporated skin using the P.L.E.A.S.E. ${ }^{\circledR}$ (Precise Laser Epidermal System) ablative laser device [4]. The epidermal skin barrier plays an important role in protecting the underlying structures and allows the passage of low molecular weight lipophilic molecules, but, restricts the passage of hydrophilic and macromolecules. For example, modes of hGH administration are restricted to subcutaneous and intramuscular injections which resulted in poor compliance including withdrawal or discontinuation of treatment by patients. Alternative administration routes such as transdermal delivery are promising; however, large peptides/ proteins including hGH cannot be delivered efficiently across the skin due to their size and hydrophilic nature. Laser-assisted microporation studied in this study creates microchannels in the skin that result in the enhancement of drug delivery. The resulting data indicate the possibility of transdermal delivery of hGH using microporation with the P.L.E.A.S.E. ${ }^{\circledR}$ technology.

The next article by Grill et al. assesses the chemopreventive efficacy of poly (lactide-co-glycolic acid) (PLGA) microparticles (MPs) loaded with curcumin in a transgenic mouse model of HER-2-positive breast cancer [5]. The paper summarizes that although sustained tissue levels of curcumin can inhibit tumorigenesis, its low potency and rapid systemic elimination are impediments to the development of a clinically translatable sustained release dosage form. Approaches such as increasing the potency or reducing the clearance rate of curcumin may allow the development of formulations suitable for cancer 
chemoprevention. Next, Owen et al. explain a versatile method for the preparation of particle-loaded microbubbles for multimodality imaging and targeted theranostic vehicles [6]. The method successfully applies to incorporate nanoparticles (NPs) into phospholipid shells to improve the theranostic potential of microbubbles. Results prove that the particle-loaded microbubbles can be produced with an appropriate size and stability needed for intravenous delivery.

A diversity of inorganic materials in nanoformulations is currently being used for biomedical applications due to their unique physicochemical properties and immense potential as drug delivery vectors. However, toxicity and a successful translation are major issues in their potential clinical application. The next few articles of this special issue discuss about the inorganic materials based drug delivery systems. Kim et al. evaluate how Pro-NPTM (biodegradable NPs containing superoxide dismutase and catalase) could provide protection against titanium dioxide $\mathrm{NPs}\left(\mathrm{TiO}_{2} \mathrm{NPs}\right)$-induced photooxidative stress in zebrafish model [7]. Results show that antioxidant Pro-NPTM exert significant protective effects mitigating the $\mathrm{TiO}_{2} \mathrm{NP}$-induced phototoxicity and could be developed as a safe/effective skin care product. Xue et al. explore the effects of PEGylation on the physicochemical properties, antioxidant activity, and biocompatibility of cerium oxide NPs (CNPs) with rod- and cube-shaped geometries [8]. CNPs represent a promising antioxidant system with potential therapeutic efficacy; however, to facilitate the clinical translation, their surface modifications via polymer grafting have been proposed to control the cellular uptake and in vivo fate. Results confirm that PEGylation does not alter the shape of CNPs and significantly reduces the protein adsorption while maintaining their antioxidant properties, cytocompatibility, and rod-shaped CNPs exhibit superior ROS scavenging properties. The work from Tyagi et al. evaluates the potential of subcutaneously administered dosing silica MPs on the surface morphology, particle size, rheology, injectability, and sustained delivery of an anti-CD40L antibody [9]. The intrinsic drawbacks of PLGA and poly(L-lactide) (PLA)-based polymeric delivery vehicles are the incomplete/burst release or instability of biologics that primarily results from the acidic environment created by the free lactic/glycolic acids. Recently, silica has been approved by the FDA as a safe, biodegradable/biocompatible material and has attracted considerable interest in drug delivery formulations (oral and topical). In comparison to polymers such as PLGA, silica degradation does not create an acidic atmosphere. The in vitro release in this study indicates less than $5 \%$ burst release whereas in vivo pharmacokinetics shows a zero-order release up to 62 days.

The oral administration is the preferred drug delivery route for patients due to its ease of administration. However, the therapeutic effectiveness of an orally administered drug is critically dependent upon its bioavailability, which is affected mainly by drug solubility. Moreover, drugs administered orally are hampered by the multidrug efflux transporter P-glycoprotein and great affinity to intestinal/ liver cytochrome P450 enzymes. Lipid-based delivery vehicles such as the self-emulsifying drug delivery systems (SEDDS) have been evaluated to enhance the bioavailability of poorly soluble drugs. These formulations, when added to an aqueous phase under moderate agitation (either prior to dosing or in situ in the gut), result in thermodynamically stable nanoemulsions. These nanoemulsions disperse readily in the gut, and their small droplet size contributes to large interfacial surface area thus a better drug absorption. However, it is difficult to predict the in vivo performance of lipid dosage forms based on conventional in vitro techniques such as cell monolayer permeability studies because of the complexity of the gastrointestinal (GI) fluids processing of lipid formulations. In the study by Sadhukha et al., the feasibility of coupling Caco-2 and MDCK monolayer permeability studies with lipolysis is evaluated as a promising in vitro technique for lipid systems [10]. Although, lipolysis is considered an important in vitro method to evaluate the in vivo performance of lipid systems, results indicate that the lipid digestion products lead to disruption of cell monolayer, and thus, it may not be appropriate to combine lipolysis with cell monolayer permeability studies. Next, the work from Almeida et al. synthesizes the self-assembled amphiphilic micelles based on chitosan (CS) and polycaprolactone (PCL) as carriers of paclitaxel to improve its intestinal pharmacokinetics profile [11]. Paclitaxel is a chemotherapeutic drug, but, has a limited oral efficiency due to its hydrophobicity. Results show that the permeability of paclitaxel is higher in Caco-2/HT29-MTX co-culture model compared with Caco-2 monolayer due to the mucoadhesive character of micelles. Therefore, it is concluded that the CS-g-PCL micelles, employed for the first time as paclitaxel carriers, may be a potential mucoadhesive system for intestinal delivery of hydrophobic anticancer agents.

The need for topical delivery led to the search for novel carriers which not only carry the molecule to the desired site but also manage their effects to produce the extended effect. Many vesicular lipid drug delivery systems have been explored to obtain the optimized formulations with tailor-made properties. Organogels have generated great interest to facilitate the transport of drugs through the topical route because of their structural and functional benefits. These are clear, thermodynamically stable, and biocompatible gel-like systems, primarily composed of hydrated phospholipids and organic liquid. Among these, lanolin-based organogels are selected by Sharma et al., to overcome the adverse effects (e.g., burning/stinging sensation) and to enhance the permeation of the drug (salicylic acid) [12]. As results say, organogels not only improve the delivery of salicylic acid but also reduce the skin irritant potential. The current findings provide an alternative for development of an effective topical 
formulation of salicylic acid for the treatment of hyperkeratotic lesions.

The application of nanoemulsions in cosmetics has been enlarged in the last decades because of several formulation advantages such as improvement in the penetration of drugs through the human skin, comparing to conventional emulsion. The work from Musazzi et al. evaluates how an oil-in-water nanoemulsion influence the in vitro skin permeation profiles of two model active ingredients (caffeine and ethyl ximenynate) with different polarity [13]. Results demonstrate that nanoemulsions significantly influence the permeation profiles of molecules as a function of their physicochemical properties. Considering such findings, it is worth reconsidering the regulatory framework based on the risk assessment of nanoemulsionbased cosmetic products.

Back of the eye diseases are the major source of vision loss especially in aging patients. While there are many medications to treat these conditions, the challenge remains to deliver them effectively with a sustained release profile and minimal side effects. Current treatments containing anti-vascular endothelial growth factor agents need frequent intravitreal administration (generally per month) due to their high clearance from ocular fluid and instability in biological environment. This generates poor patient compliance accompanied by numerous side effects and an increase in the cost of the therapy. As a result, the work from Agrahari et al. synthesizes novel tailor-made pentablock copolymer-based composite nanoformulations for a long-term ocular delivery of therapeutic molecules [14]. The investigation compares the effect of hydrodynamic diameter of macromolecules, polymer arrangements (various ratios and molecular weight), and hydrophobicity on the release profile of macromolecules. The outcome of this study suggests that the pentablock copolymer-based composite nanosystems are a promising platform for the posterior and anterior segment eye diseases.

The sense of hearing is essential, and its dysfunctions can strongly impact on the quality of life. In this context, the cochlea plays a fundamental role in the transformation of the airborne sound waves into electrical signals, which can be processed by the brain. However, several diseases and external stimuli can damage the sensorineural structures of the cochlea, inducing progressive hearing dysfunctions until deafness. Several innovative drug delivery systems such as in situ gels, nanocarriers, and nanocarrier-loaded hydrogels are designed for inner ear drug delivery approaches, and their efficacy has been proven in cochlear models. Based on these premises, the review by Musazzi et al. highlights and discusses the critical aspects of the cochlear drug delivery, the anatomy and physiology of the inner ear, and the critical considerations need to be taken while designing the delivery systems [15].

Drug delivery research for human use has been focused on the use of novel systems and other expensive approaches that may not be applicable in animal health research. There are currently immense efforts being performed into the development of products for veterinary applications; however, translating research into a veterinary product is challenging. A further challenge is how to best use limited resources during translation, when to move from the laboratory to animal, and how many animals to use in early/pilot studies. The manuscript from Tucker et al. describes a translational journey to emphasize that translation is not straight forward and demonstrate the gathering of opportunistic data to inform the process [16]. Translation of a ready-to-use antibiotic formulation in an oily vehicle for use as an intramuscular injection in dairy cows for the treatment of bacterial mastitis is described in this work.

In summary, the special issue, "Novel Drug Delivery Systems, Devices, and Fabrication Methods", incorporates advances in novel drug delivery systems for various indications while highlighting their development, application, and translational challenges. We therefore hope that our readers find this issue comprehensive and highly resourceful for the development of delivery systems. This special issue would not have been possible without the timely submission and revision by the contributors, critical evaluations by the reviewers, the assistance provided by the Drug Delivery and Translational Research (DDTR) journal, and Springer editorial staff members. They all ensured that the manuscripts are carefully evaluated, processed, reviewed, and published at high standards. A special thanks to Prof. Vinod Labhasetwar, Editor-in-Chief, for giving the opportunity to develop this special issue and for his constant support throughout the process. We expect that the scientific community engaged in the drug delivery research and translational approaches may find this special issue an interesting addition to the available literature.

\section{Compliance with ethical standards}

Conflict of interest The authors declare that they have no conflict of interest.

\section{References}

1. Agrahari V, Agrahari V, Mitra AK. Nanocarrier fabrication and macromolecule drug delivery: challenges and opportunities. Ther Deliv. 2016;7(4):257-78. https://doi.org/10.4155/tde-2015-0012.

2. Agrahari V, Hiremath P. Challenges associated and approaches for successful translation of nanomedicines into commercial products. Nanomedicine (Lond). 2017;12(8):819-23. https://doi.org/10. 2217/nnm-2017-0039.

3. Quinn HL, Hughes CM, Donnelly RF. In vivo and qualitative studies investigating the translational potential of microneedles for use in the older population. Drug Deliv Transl Res. 2017; https://doi. org/10.1007/s13346-017-0393-4.

4. Song Y, Hemmady K, Puri A, Banga AK. Transdermal delivery of human growth hormone via laser-generated micropores. Drug Deliv Transl Res. 2017; https://doi.org/10.1007/s13346-017-0370-y. 
5. Grill AE, Shahani K, Koniar B, Panyam J. Chemopreventive efficacy of curcumin-loaded PLGA microparticles in a transgenic mouse model of HER-2-positive breast cancer. Drug Deliv Transl Res. 2017; https://doi.org/10.1007/s13346-017-0377-4.

6. Owen J, Crake C, Lee JY, Carugo D, Beguin E, Khrapitchev AA, et al. A versatile method for the preparation of particle-loaded microbubbles for multimodality imaging and targeted drug delivery. Drug Deliv Transl Res. 2017; https://doi.org/10.1007/s13346017-0366-7.

7. Kim MS, Stees M, Karuturi BVK, Vijayaraghavalu S, Peterson RE, Madsen GL, et al. Pro-NP protect against TiO2 nanoparticleinduced phototoxicity in zebrafish model: exploring potential application for skin care. Drug Deliv Transl Res. 2017;7(3):372-82. https://doi.org/10.1007/s13346-017-0374-7.

8. Xue Y, Balmuri SR, Patel A, Sant V, Sant S. Synthesis, physicochemical characterization, and antioxidant effect of PEGylated cerium oxide nanoparticles. Drug Deliv Transl Res. 2017; https://doi. org/10.1007/s13346-017-0396-1.

9. Tyagi P, Koskinen M, Mikkola J, Leino L, Schwarz A. Silica microparticles for sustained zero-order release of an anti-CD40L antibody. Drug Deliv Transl Res. 2017; https://doi.org/10.1007/ s13346-017-0408-1.

10. Sadhukha T, Layek B, Prabha S. Incorporation of lipolysis in monolayer permeability studies of lipid-based oral drug delivery systems. Drug Deliv Transl Res. 2017; https://doi.org/10.1007/s13346-0170383-6.
11. Almeida A, Silva D, Goncalves V, Sarmento B. Synthesis and characterization of chitosan-grafted-polycaprolactone micelles for modulate intestinal paclitaxel delivery. Drug Deliv Transl Res. 2017; https://doi.org/10.1007/s13346-017-0357-8.

12. Sharma G, Devi N, Thakur K, Jain A, Katare OP. Lanolin-based organogel of salicylic acid: evidences of better dermatokinetic profile in imiquimod-induced keratolytic therapy in BALB/c mice model. Drug Deliv Transl Res. 2017;7(5):683-94. https://doi.org/ 10.1007/s13346-017-0364-9.

13. Musazzi UM, Franze S, Minghetti P, Casiraghi A. Emulsion versus nanoemulsion: how much is the formulative shift critical for a cosmetic product? Drug Deliv Transl Res. 2017; https://doi.org/10. 1007/s13346-017-0390-7.

14. Agrahari V, Patel SP, Dhall N, Aulgur Z, Thukral S, Yang X, et al. Nanoparticles in thermosensitive gel based composite nanosystem for ocular diseases. Drug Deliv Transl Res. 2017. https://doi.org/10. 1007/s13346-017-0435-y.

15. Musazzi UM, Franze S, Cilurzo F. Innovative pharmaceutical approaches for the management of inner ear disorders. Drug Deliv Transl Res. 2017; https://doi.org/10.1007/s13346-0170384-5.

16. Tucker IG, Jain R, Alawi F, Nanjan K, Bork O. Translational studies on a ready-to-use intramuscular injection of penethamate for bovine mastitis. Drug Deliv Transl Res. 2017; https://doi.org/10. 1007/s13346-017-0388-1. 